\title{
Simulation of surface effects in energy dissipation of ultrahighfrequency (UHF) nanocantilevers
}

\author{
Kun Yan, Ai-Kah Soh \\ Dept. of Mechanical Engineering, Univ. of Hong Kong, Pokfulam, Hong Kong, PRC
}

\begin{abstract}
Devices composed of nanoelectromechanical systems (NEMS) possess distinguished properties which make them quite suitable for a variety of applications including ultra-high-frequency (UHF) resonators. However, most GHz resonators have low quality factor even though it has been well above $10^{3} \sim 10^{5}$ for very-high-frequency (VHF) microresonators. The motivation for our investigation of single crystal silicon nanoresonator arises from both its technological importance and its extraordinary surface effects. Our simulation results show that the quality factor decreased in a nearly linear manner as the surface area to volume ratio (SVR) was increased, which suggests that surface losses play a significant role in determining the quality factor of nanoresonators.
\end{abstract}

Keywords: quality factor, nanoresonator, surface area to volume ratio

\section{INTRODUCTION}

Great efforts have been made to develop novel synthesis techniques for fabrication of morphologically and structurally controlled nanomaterials. It is also essential to characterize the nanostructures and their collective properties in varying working conditions. Electron-beam lithography and nanomachining now enable semiconductor nanostructures to be fabricated under 10 nanometers, and the devices composed of these nanostructures possess distinguished properties, such as ultra-high integration level, approximately $10^{12}$ elements per square centimeter, and fundamental frequencies in microwave range $(\sim 100 \mathrm{GHz}){ }^{1}$, which make them quite suitable for application as force sensors, chemical sensors, biological sensors, and ultra-high-frequency (UHF) resonators. However, the quality factor ( $Q$ factor) of most UHF nanoresonators is usually limited to $1000^{2,3}$, which is extremely low when compared to the $10^{3} \sim 10^{5} Q$ factor of the very-high frequency (VHF) microresonators ${ }^{4,5}$. It was proposed that surface losses may play a significant role in determining the quality factor. Recent experiments have shown that the quality factor in many devices decreased in a linear manner as the surface-to-volume ratio (SVR) was increased ${ }^{3}$. And in another investigation, the surface-related mechanism was also believed to cause the periodical variation of $Q$ factor of a $50 \mathrm{~nm}$ thick single crystal Si cantilever with its crystallographic orientation ${ }^{6}$. First-principles calculations are widely employed because of their high accuracy, and the results obtained are often used for comparison with experimental results as well as for improvement of the semi-empirical interatomic potentials As one of the simulation tools for studying both the static and dynamic properties of nanomaterials, molecular dynamics (MD) method has advantages over first-principles calculations because they can handle much larger systems, from which the macroscopic properties can be drawn from the results.

Apart from being the basic material for integrated circuit (IC) technology during the past few decades, silicon is extensively employed for the fabrication of NEMS. In the present study, MD simulations are employed to investigate the energy dissipation of silicon-based nanoelectromechanical systems (NEMS). Ultrathin silicon cantilevers with low aspect ratio (length to thickness) and extremely high surface area to volume ratio will be characterized by the resonant frequency based on under-damped flexural oscillations. Both the resonant frequency and quality factor of these $\mathrm{Si}$ nanocantilevers will be related to their surface area to volume ratio.

Modeling, Signal Processing, and Control for Smart Structures 2008, edited by Douglas K. Lindner Proc. of SPIE Vol. 6926, 69260X, (2008) · 0277-786X/08/\$18 - doi: 10.1117/12.775386 


\section{METHODOLOGY}

Most nanoresonators are typically free standing samples vibrating predominantly either in flexure or torsion. In the present simulation, silicon nanocantilevers with five different thicknesses and eight different aspect ratios (length to thickness) are considered. In accordance with Bernoulli-Euler theory, the differential equation for the free transverse vibration $z(x, t)$ of a cantilever is given by:

$$
\frac{E I}{m} \cdot \nabla^{4} z+\frac{\xi}{m} \dot{z}+\ddot{z}=0
$$

where $E, I, m$ and $\xi$ denote the Young's modulus, moment of inertia, mass per unit length and damping parameter per unit length, respectively. For a cantilever with one end clamped, the necessary boundary conditions to solve the above equation are $z=\frac{\partial z}{\partial x}=0$ for the clamped end at $x=L$, and $\frac{\partial^{2} z}{\partial x^{2}}=\frac{\partial^{3} z}{\partial x^{3}}=0$ for the free end at $x=0$, as shown in figure 1.

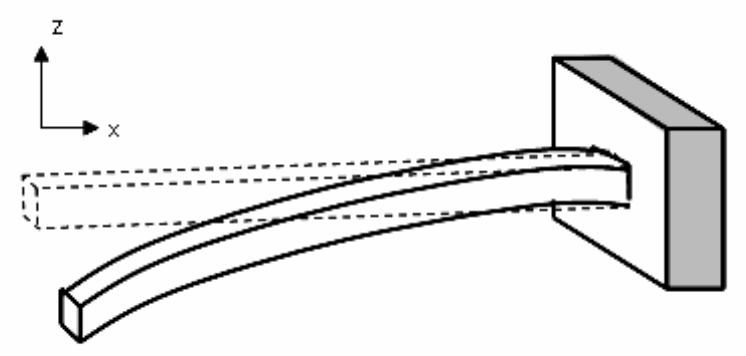

Fig.1 Simulation of nanocantilever oscillations

Simulations of the vibrational behavior of Si nanocantilevers, which have thicknesses between $3 \mathrm{~nm}$ and $10 \mathrm{~nm}$ and their lengths adjusted according to thickness via the aspect ratio $\alpha(=L / d)$, are carried out. The configuration of a typical nanocantilever is shown in figure 2 .

The empirical Stillinger-Weber (SW) many-body potential is employed for interatomic interactions, and Newton's equations of motion are calculated with a time step of $0.38 \mathrm{fs}$ (femosecond) in a Gear's predictor-corrector algorithm. At least sixteen empirical potentials have been developed for silicon to date ${ }^{7},{ }^{8}$. However, the empirical nature of these potentials accounts partially for the controversy on which potential gives a better description of the covalent bonding. The SW interatomic potential is by far the most widely used potential among those developed for silicon. It has been used to study lattice dynamics, bulk point defects, surface diffusions and reconstructions, crystal-melt interfaces, pulsed melting of surfaces, and epitaxial growth from vapor, etc.

The SW potential is a short-ranged cluster potential, which models bonding using the classical two- and three-body potentials, for which only the first-neighbor is taken into account in the bond interaction term. The interaction cutoff in 
the SW potential is set to be $3.77 \AA$, which is between the distance of the first-neighbor and that of the second-neighbor. The radial distribution function (RDF) calculated for crystalline $\mathrm{Si}$ at room temperature is plotted in figure 3, reproducing well the experiments.

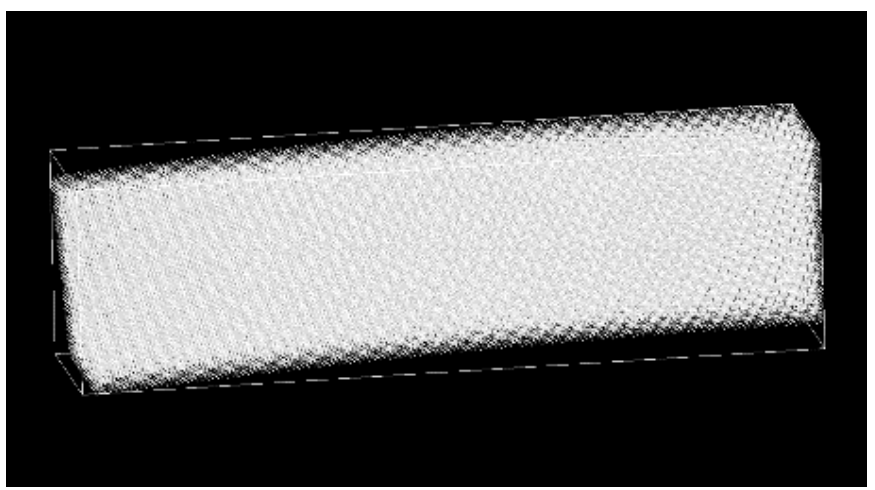

Fig.2 Initial configuration of a typical Si nanocantilever

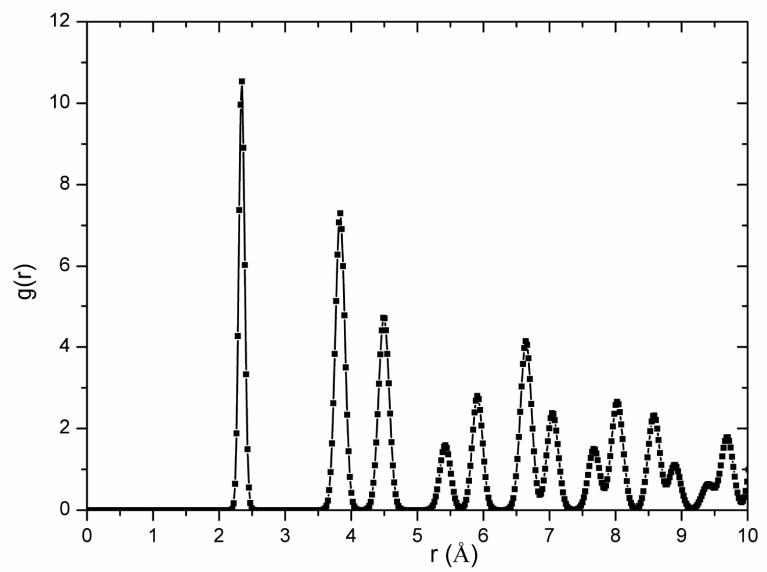

Fig. 3 Radial distribution function (RDF) of the Si sample in the range of distance permitted by simulation size

\section{RESULTS AND DISCUSSIONS}

\subsection{Damping process}

In order to consider the dominance of surface effects on the anelastic behavior of the nanocantilevers, the surface area to volume ratio (SVR) is selected for measurement. The SVR of a nanocantilever with thickness $d$ and aspect ratio $\alpha$ is given by $S V R=\left(4+2 \alpha^{-1}\right) d^{-1}$. Given an aspect ratio of the nanocantilever, the SVR is inversely proportional to the thickness. 
Two distinct anelastic processes can be observed for nanocantilevers with different SVRs. For instance, figure 4(a) shows that the mechanical oscillation amplitude of the $4.6 \mathrm{~nm}$ thick nanocantilevers with varying aspect ratio degenerates rapidly into an erratic motion without showing any trend of exponential decay. In contrast, the $9.215 \mathrm{~nm}$ thick nanocantilevers with the same range of aspect ratio illustrate a regular anelastic behavior, as shown in figure 4(b), in which the oscillation amplitudes exhibit the trend of exponential decay.

It seems that the anelastic behavior of the nanocantilevers with larger SVR may deviate from the regular damping mode illustrated by bulk materials. This is because the surface effects rather than bulk effects dominate, in which case the energy is transferred from the fundamental mode into other modes. Our results show that as the size of the nanocantilever shrinks to a stage where SVR exceeds $1.2 \mathrm{~nm}^{-1}$, the damping of the oscillation is anomalous.
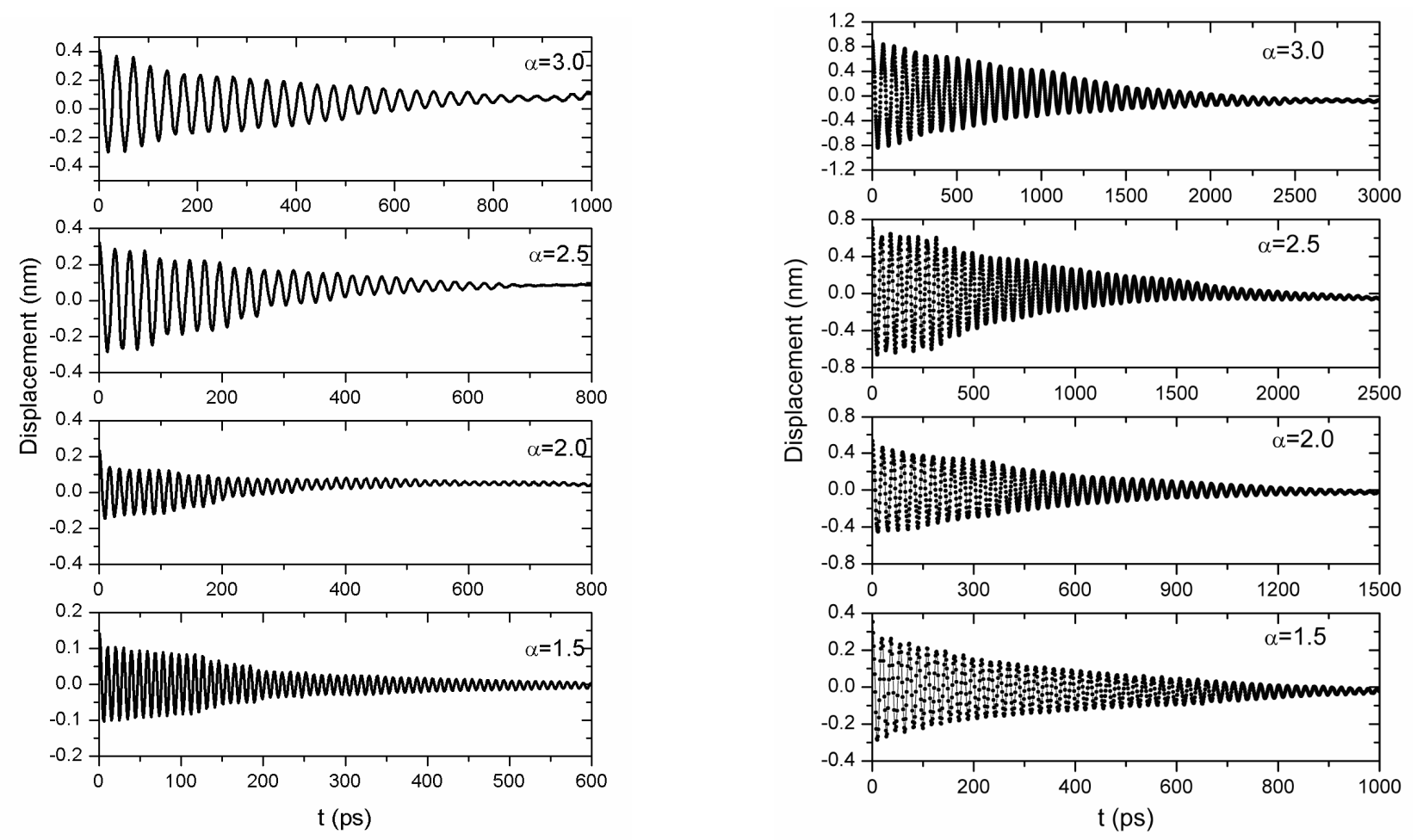

(b)

Fig. 4 Flexural displacement as a function of time for nanocantilevers with different thicknesses: (a) $4.6 \mathrm{~nm}$; and (b) $9.215 \mathrm{~nm}$

\subsection{Resonant frequency}

For undamped oscillation, i.e., $\xi=0$, the resonance frequency for a uniform cantilever is given by:

$$
\omega=\frac{\lambda^{2}}{L^{2}} \sqrt{\frac{E I}{m}}
$$

where $\rho$ is the material density. The frequency parameter for the first mode is given by $\lambda=1.875$. 
The above equation can also be expressed as

$$
\omega=\frac{\lambda^{2} d}{L^{2}} \sqrt{\frac{E}{12 \rho}}=c_{0} \beta
$$

which indicates that the resonant frequency is related to the mechanical parameter $c_{0}=\lambda^{2} \sqrt{\frac{E}{12 \rho}}$ and geometric parameter $\beta=\frac{d}{L^{2}}$, where $d$ is the cantilever thickness.

In the case of under-damped oscillation, i.e., $0<\xi<1$, the resonant frequency deviates from the undamped resonant frequency,

$$
\omega^{d}=\omega \sqrt{1-\xi^{2}}=c_{1} \beta
$$

where $c_{1}=\sqrt{1-\xi^{2}} c_{0}$. Therefore, Young's modulus can be determined from $c_{1}$, which can be calculated from the resonant frequency as a function of geometry parameter $\beta$.

Figure 5 shows that the resonant frequencies of the nanocantilevers obtained from our simulations increase almost linearly with increasing geometric factor $\beta$ for various thicknesses between $3 \mathrm{~nm}$ and $10 \mathrm{~nm}$, which are in exact agreement with equation (4) in which the resonant frequency is determined based on the continuum theory. Note that the lack of experimental and computational data for sub-10nm nanocantilevers makes it impossible for us to further confirm our simulation results.

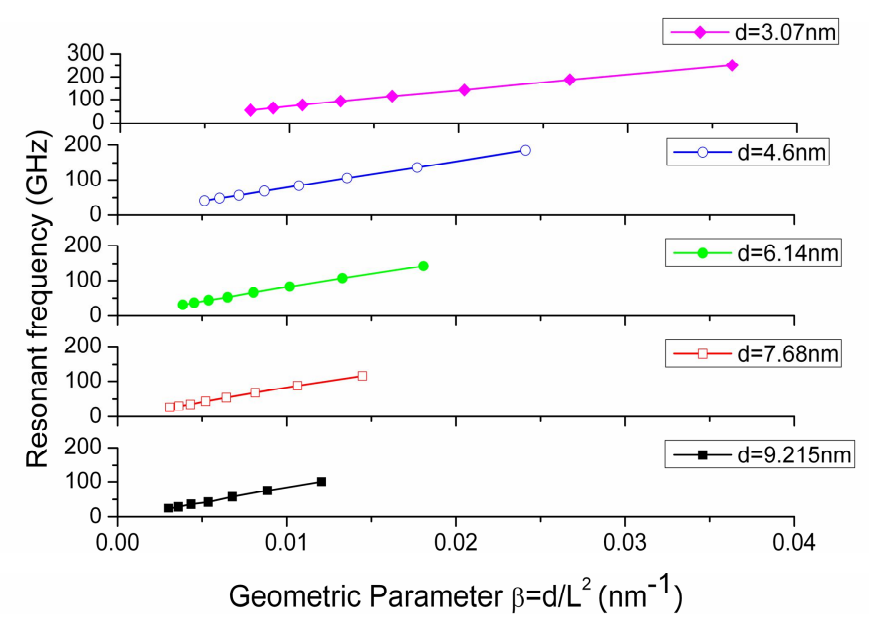

Fig. 5 Plots of resonant frequencies calculated from MD simulations for different geometries of nanocantilevers. 


\subsection{Energy dissipation}

The amplitude of underdamped oscillation $A(t)$ can be expressed as,

$$
A(t)=A^{0} \exp (-\xi \omega \cdot t)=A^{0} \exp (-t / \tau)
$$

where $\tau$ is the decay constant of the amplitude. For very weak damping ( $\xi \rightarrow 0$ ), we have,

$$
\xi \tau=\frac{1}{\omega} \cong \frac{1}{\omega^{d}}
$$

Thus, the damping ratio is related to the oscillation period $T$ and the decay constant $\tau$ as follows:

$$
\xi=\frac{1}{2 \pi}\left(\frac{T}{\tau}\right)
$$

Since the decay constant $\tau$ can be calculated from the exponential decay fit of the amplitude of oscillation $A(t)$, the quality factor $Q$, which is the inversion of energy dissipation (or internal friction), can be evaluated from the above expression as follows:

$$
Q=\frac{\omega^{d}}{2 \xi \omega^{0}} \approx \frac{1}{2 \xi}=\pi \frac{\tau}{T}
$$

The Q factor deduced from the current simulations is between 40 and 70, which is comparable with the experimental Q factor of oscillating nanotubes with extremely large SVR ${ }^{9}$. The variation of the predicted dissipation at resonance with respect to the geometries of nanocantilever is illustrated in figure 6. For a given aspect ratio $\alpha$, the quality factor decreases with increasing resonant frequency. Furthermore, the plots of quality factor versus SVR, as shown in figure 7 , illustrate that when the SVR of the Si nanocantilever is increased from 0.5 to $1.1 \mathrm{~nm}^{-1}$, the reduction of quality factor is nearly $28 \%$.

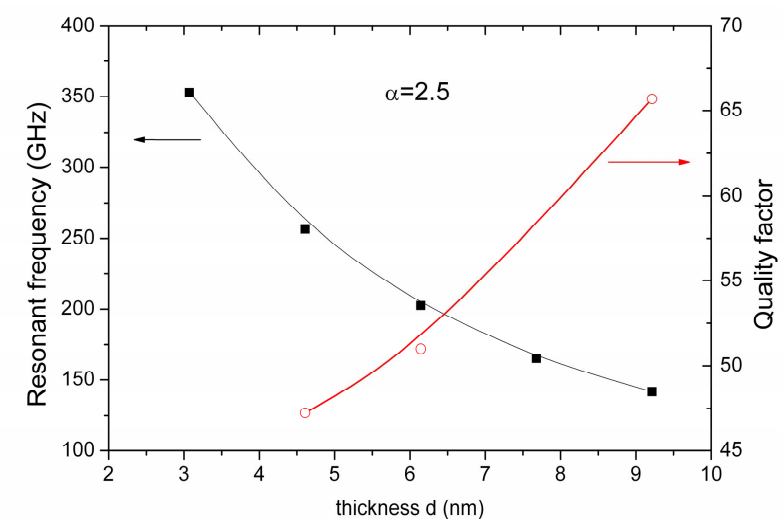

(a) 


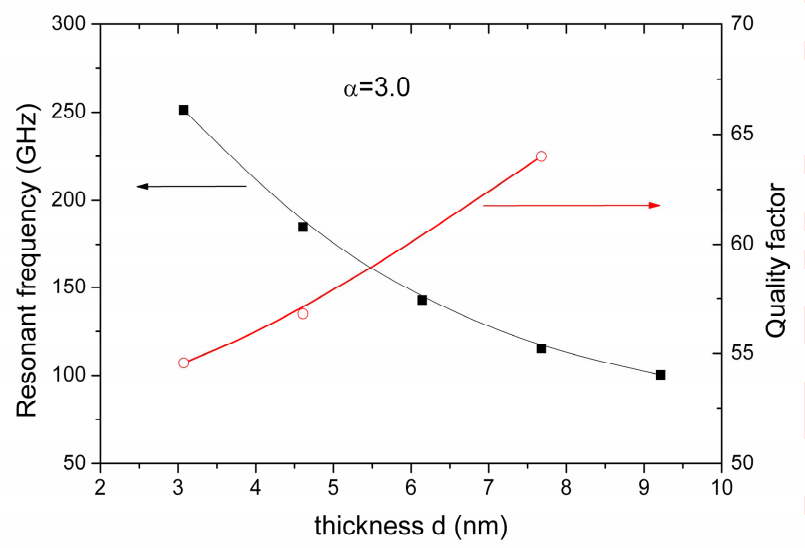

(b)

Fig. 6 Dissipations as well as resonant frequencies are plotted for different geometries of Si nanocantilevers

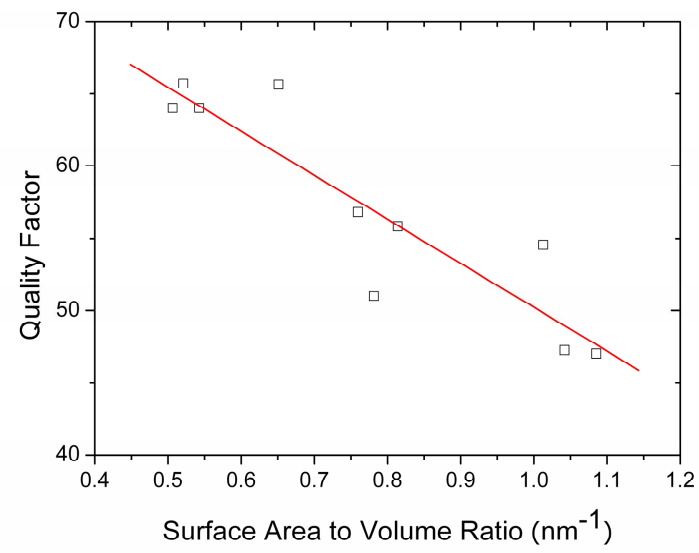

Fig. 7 Dissipation versus surface area to volume ratio of Si nanocantilevers

\section{CONCLUSIONS}

Molecular dynamics (MD) simulations are employed to analyze the anelastic behavior of Si nanocantilever with extremely large surface area to volume ratio (SVR). The quality factors of nanocantilevers with different geometries are calculated via analyzing the displacement of the mechanical oscillation of these nanocantilevers. The results show that the quality factor decreases by approximately $28 \%$ when SVR is increased from 0.5 to $1.1 \mathrm{~nm}^{-1}$. Moreover, when the SVR exceeds $1.2 \mathrm{~nm}^{-1}$, the amplitude decay of the nanocantilever does not exhibit the same regular exponential trend observed in bulk materials. 


\section{ACKNOWLEDGEMENTS}

Support from the Research Grants Council of the Hong Kong Special Administrative Region, China (Project No. HKU $7195 / 04 E$ ) is acknowledged.

\section{REFERENCES}

1. T. Rueckes, K. Kim, E. Joslevich and G. Y. Tseng, "Carbon nanotube-based nonvolatile random access memory for molecular computing," Science 289, (2000).

2. R. E. Rudd, "Coarse-Grained molecular dynamics and multiscale modeling of NEMS resonators," in Int. Conf. Comput. Nanosci., (2002).

3. P. Mohanty, D. A. Harrington and K. L. Ekinci, "Intrinsic dissipation in high-frequency micromechanical resonators," Phys. Rev. B 66, 15 (2002).

4. M. L. Roukes, "Nanoelectromechanical systems face the future,," Phys. World 14, (2001).

5. J. Yang, T. Ono and M. Esashi, "Energy dissipation in submicrometer thick single-crystal silicon cantilevers," J.

MicroElectroMech. Sys. 11, 9 (2002).

6. D. F. Wang, T. Ono and M. Esashi, "Crystallographic influence on nanomechanics of (100)-oriented silicon resonators," Appl. Phy. Lett. 83, 3 (2003).

7. G. Ackland, "Semiempirical model of covalent bonding in silicon," Phys. Rev. B 40, 5 (1989).

8. H. Balamane, T. Halicioglu and W. A. Tiller, "Comparative study of silicon empirical interatomic potentials," Phys. Rev. B 46, 30 (1992).

9. V. Sazonova, Y Yaishi, H. Ustunel and D. Roundy, "A tunable carbon nanotube electromechanical osicllator," Nature 431, 4 (2004). 